\title{
Algebraic Versus Geometric Thought and Expression in the Early Calculus
}

\author{
Viktor Blåsjö
}

\section{Contents}

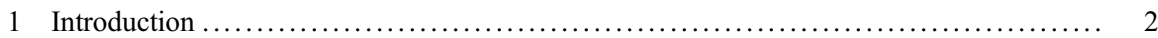

2 Absence of Trigonometric Functions in Early Calculus ........................... 2

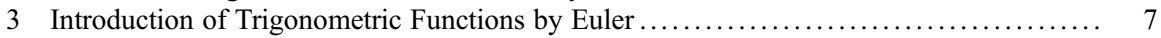

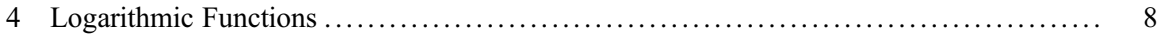

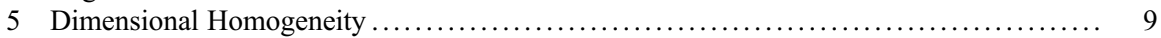

6 Did Barrow Prove the Fundamental Theorem of Calculus? ........................ 11

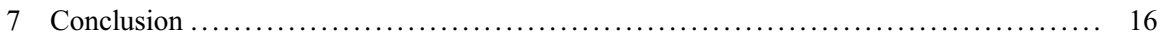

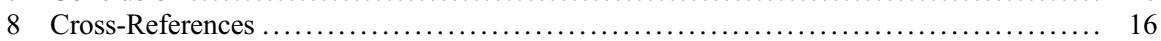

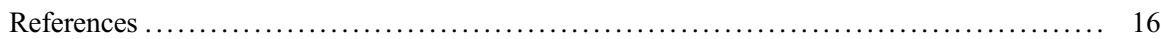

\section{Abstract}

The language of the early calculus was much more geometrical than the analytic and algebraic style that was pioneered by Euler and still dominates today. For instance, functions such as $\sin (x)$ and $\log (x)$ were largely absent from the early calculus, with geometric paraphrases used in their place. From a modern standpoint, one may be inclined to assume that the eventual triumph of the more analytic perspective was a straightforward case of progress, and that the geometric aspects of the early calculus were a historical artifact ultimately hampering this development. Interestingly, however, in private notes, the pioneers of the calculus showed a readiness to disregard traditionalism and operate freely in a more protomodern style than they allowed themselves in their publications. This suggests that the adherence to the geometrical mode in published works was a deliberate choice selected with full awareness of the analytic alternative. Indeed, the geometrical paradigm was no mere blind conservatism or lip service to classical foundations; rather, it arguably had genuine merits, for example, as an intuitionboosting heuristic strategy.

\footnotetext{
V. Blåsjö

Universiteit Utrecht, Utrecht, The Netherlands

e-mail: V.N.E.Blasjo@uu.nl
} 
This aspect of the early calculus can serve as a case study that illuminates the relation between official expression and informal thought in mathematics more generally. For one thing, it complicates the common historiographic assumption that fidelity to historical thought is best achieved by following the original text's mode of expression as closely as possible.

\section{Keywords}

Leibniz $\cdot$ Newton $\cdot$ Barrow $\cdot$ Euler $\cdot$ History of infinitesimal calculus · Fundamental theorem of calculus $\cdot$ Dimensional homogeneity $\cdot$ Anachronism $\cdot$ Historiography

\section{Introduction}

When studying a historical mathematical text, should we attempt to interpret it in terms of later mathematical concepts? Rampant anachronism can be a mark of insensitivity and a closed mind; it can drown out subtleties and rob us of one of the greatest rewards of studying history in the first place - that of expanding our own mindset and perspective. But banning all anachronistic analysis as illegitimate risks making historical scholarship conceptually impoverished and pathologically preoccupied with incidental details. The question is ultimately one that not only the historian but also the philosopher of mathematical practice must grapple with: Can a mathematician's written word be taken as constitutive of their underlying thought?

The early development of the infinitesimal calculus in the late seventeenth century provides a rich store of case studies for these questions. In addition to interesting differences between the original and modern forms of the calculus, we are fortunate that extensive personal manuscripts by the pioneers of the calculus have been preserved (and in many cases recently published for the first time), helping us further triangulate the relations between thought and expression, tradition and innovation.

\section{Absence of Trigonometric Functions in Early Calculus}

The early practitioners of the calculus virtually never used any trigonometric expres$\operatorname{sions} \operatorname{such}$ as $\sin (x)$ in their calculus. Neither Newton nor Leibniz ever wrote $\sin (x)$ in any calculus formula in any of their published works. They and many others did write expressions like this in purely geometric contexts, such as referring to sine tables. But they never treated $\sin (x)$ or any other trigonometric expression as a function in a calculus context. Unlike us, they did not see the differentiation and anti-differentiation of such expressions as among the most basic and commonly used calculation rules. They never described curves or solutions to differential equations in terms of such expressions. 
Fig. 1 Uniqueness of circular orbit through a given point in a $\mathbf{F}=-k \mathbf{r}$ force field

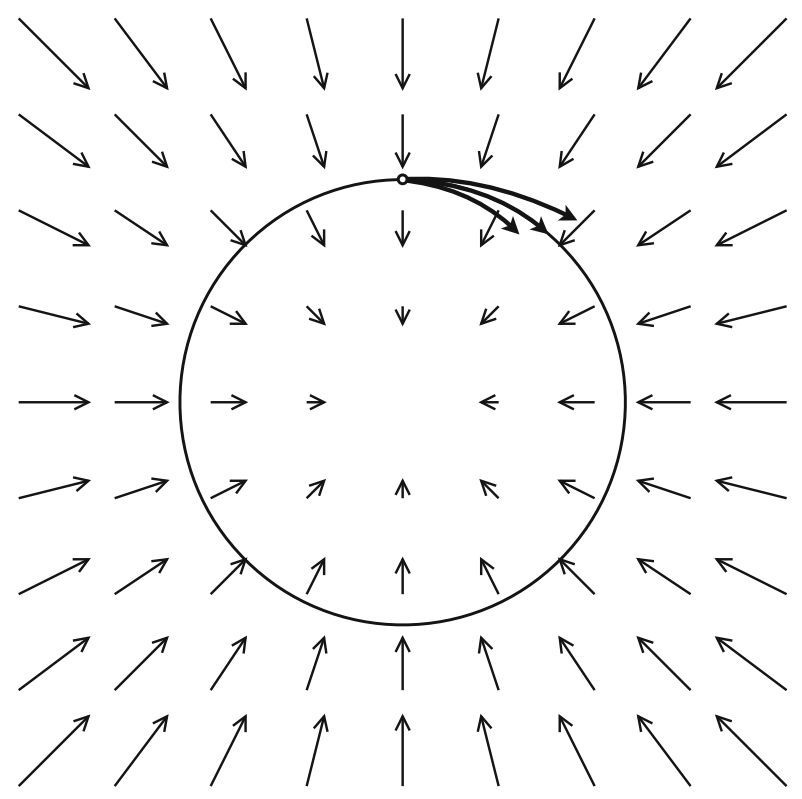

How can this be, when any modern calculus textbook is packed with those kinds of uses of trigonometric functions? How can one have a functioning calculus without this tool? In fact, the early practitioners of the calculus were not in any way handicapped by this choice. Let us look at a few examples of how they managed without trigonometric functions. Let us consider some examples of problems where the treatment in a modern calculus textbook seems most essentially based on trigonometric functions, and see how Newton and Leibniz managed to treat these problems just as well, if not better, in other terms.

Simple harmonic motion - such as the bobbing motion of a weight on a spring - is a prototype example of a physical phenomenon described by a sine curve. The harmonic motion of a spring follows from Hooke's law that force is proportional to extension, $F \propto x$. Combined with Newton's law $F=m a=m \ddot{x}$, this means that the differential equation for the motion of the weight on the spring is $x=-k \ddot{x}$. Today, we would express the solutions to this equation in terms of sine and cosine functions. Yet in the seventeenth century people managed without it.

Newton dealt with this problem. Let's see how he did it. Newton considered a more general situation: motion in a force field where the force is proportional to distance from the origin, and directed toward the origin. This is analogous to a gravitational force field, but with a different force law. For any given starting position, a body can be launched into a circular orbit if it is given just the right sideways velocity. If the speed is increased or diminished by any amount, this would cause a deviation from the circular path (Fig. 1). Thus a specific orbital speed is naturally and intrinsically associated with this force field and this starting position. 
Table 1 Behavior of a body being dropped from $(x, y)=(0, R)$ in a $\mathbf{F}=-k \mathbf{r}$ force field

\begin{tabular}{l|l|l|l}
\hline & Position at given time & $\begin{array}{l}\text { Time to reach given } \\
\text { position }\end{array}$ & Speed at given position \\
\hline $\begin{array}{l}\text { In terms of } \\
\text { formulas }\end{array}$ & $y(t)=R \cos (\sqrt{k} t)$ & $t(y)=\frac{\arccos \left(\frac{y}{k}\right)}{\sqrt{k}}$ & $\begin{array}{r}\dot{y}(y)= \\
-\sqrt{k} R \sin \left(\arccos \left(\frac{y}{R}\right)\right)\end{array}$ \\
\hline $\begin{array}{l}\text { In terms of the } \\
\text { associated } \\
\text { circular-orbit } \\
\text { motion }\end{array}$ & $\begin{array}{l}y \text {-position of the } \\
\text { orbital motion at that } \\
\text { time }\end{array}$ & $\begin{array}{l}\text { Time in which orbital } \\
\text { motion reaches that } y\end{array}$ & $\begin{array}{l}y \text {-direction speed of } \\
\text { orbital motion at that } y\end{array}$ \\
\hline
\end{tabular}

By taking this speed as a reference, one can describe harmonic motion in this field in a natural and precise way without the need for formulas.

This is precisely what Newton does in the Principia, Book I, Prop. 38, which is about the simple harmonic motion of a dropped object in a $F \propto r$ force field. He expresses everything in terms of the associated circular-orbit motion. Table 1 puts this approach side by side with the modern approach using formulas. Newton's way has a number of advantages. For instance, the equivalence of the three different results shown is immediately evident from the geometric descriptions but takes some algebra to derive from the formulas. Furthermore, Newton's mode of expression uses language intrinsic to the system itself: the naturally associated orbital motion. The formula approach, on the other hand, becomes ugly and opaque precisely because it uses extrinsic frames of reference. The constants $k$ and $R$ depend on our choice of units of force and position, and the trigonometric functions assume a unit radius and hence have to be scaled in various ways. Those are conventions that are external to the specific scenario at hand, yet they dominate the formulas. This is why the formulas are opaque and hide the simple dynamics of the system: the formulas are primarily focused on accommodating the system to a fixed external reference frame rather than on describing the system in the clearest and most intuitive terms.

Another example where modern calculus textbooks make trigonometric functions seem indispensable is the cycloid. "The only convenient way of representing a cycloid is by means of parametric equations," one standard textbook proclaims (Simmons 1996, 592). In the discussion that follows, the expressions sin and cos occur 40 times in the space of two pages, making the cycloid a good candidate for the most trig-intensive example in the standard calculus repertoire.

Leibniz did not think this was "the only convenient way of representing a cycloid." In fact, the very opposite is the case: Leibniz explicitly and repeatedly used the cycloid as his prime example of how "perfectly" the calculus could represent curves, but his representation is very different from the "only convenient" one of modern textbooks. In his very first paper on the integral calculus, he stresses that his "equation expresses the relation between the ordinate $y$ and the abscissa $x$ 
Fig. 2 The cycloid

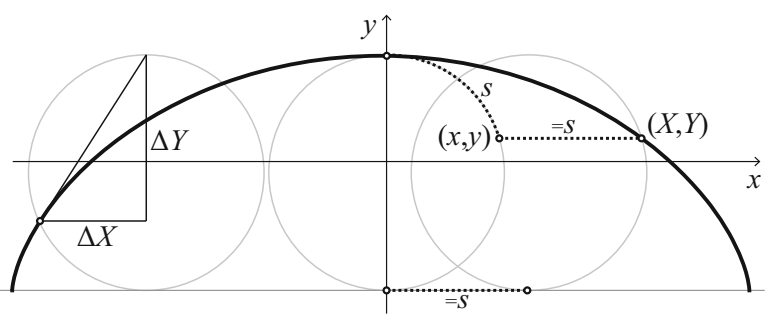

perfectly" and "from it all the properties of the cycloid can be derived" (Blåsjö 2017, 65).

Leibniz's "perfect" way of expressing the cycloid involves no trigonometry and no parametric equations. It is based instead on a simple geometrical property of the cycloid shown in Fig. 2: The three dashed lengths are all equal, since the arc $s$ measures both the amount of rotation needed to bring the tracing point from the top position down to $(x, y)$, and the amount of arc that has been in contact with the ground during this rotation, that is, the distance traveled, or the horizontal displacement $X-x$. Therefore an equation for the cycloid is $X=x+s$. This equation can be expanded, if desired, by expressing $x$ using the Pythagorean Theorem and $s$ as an arc-length integral. Assuming that the generating circle has unit radius, this gives

$$
X(y)=x+s=\sqrt{1-y^{2}}+\int_{y}^{1} \sqrt{1+\left(\frac{d x}{d y}\right)^{2}} d y=\sqrt{1-y^{2}}+\int_{y}^{1} \frac{d y}{\sqrt{1-y^{2}}}
$$

This is essentially Leibniz's "perfect" equation for the cycloid, except he centers his coordinate system on our $y=-1$, so he gets the equivalent $\sqrt{2 y-y^{2}}+$ $\int d y / \sqrt{2 y-y^{2}}$ instead.

Leibniz does not spell out how "all the properties of the cycloid can be derived" from this equation, but this can indeed be done. Let us consider how such an approach can lead to the same results that are derived using trigonometric expressions in modern textbooks such as Simmons (1996).

What is the area $A$ of the cycloid, in terms of the area $a$ of the generating circle? Integrating the equation $X=x+s$, we get

$$
\begin{aligned}
\frac{A}{2} & =\int_{-1}^{1} X d y=\int_{-1}^{1} x d y+\int_{-1}^{1} s d y=\frac{a}{2}+[y s]_{y=-1}^{y=1}-\int_{-1}^{1} y \frac{d s}{d y} d y=\frac{3 a}{2}+\int_{0}^{\pi} y d s \\
& =\frac{3 a}{2}+[x]_{s=0}^{s=\pi}=\frac{3 a}{2}
\end{aligned}
$$

so the area of the cycloid is three times the area of the generating circle, $A=3 a$. We see that Leibniz's equation for the cycloid indeed lends itself very well to calculation. The steps of this calculation do not correspond to the standard modern solution and 
arguably compare favorably to it. A key ingredient of the modern proof is the use of a trigonometric addition formula to express $\cos ^{2}(\theta)$ in terms of $\cos (2 \theta)$. The Leibnizian proof does not need this technical machinery. The modern approach ultimately reduces the integral to the standard antiderivative $\int \cos (\theta) d \theta=\sin (\theta)$. The Leibnizian approach does the same thing in the step $\int y d s=x$, which is nothing but a different way of saying $\int \cos (\theta) d \theta=\sin (\theta)$. Thus, the Leibnizian calculus contains the exact equivalent of our standard trigonometric derivatives and antiderivatives, but expresses them in other terms.

What is the tangent of the cycloid at any given point? In fact, the tangent passes through the top point of the rolling circle. This too is derived with quite elaborate trigonometric machinery in Simmons (1996) and other standard textbooks. From Leibniz's equation, we can get this result without any trigonometry. For we find that

$$
\begin{aligned}
\frac{d}{d y} X & =\frac{d}{d y}(x+s)=\frac{d}{d y}\left(\sqrt{1-y^{2}}+\int_{y}^{1} \frac{d y}{\sqrt{1-y^{2}}}\right) \\
& =\frac{-y}{\sqrt{1-y^{2}}}-\frac{1}{\sqrt{1-y^{2}}}=\frac{-(1+y)}{\sqrt{1-y^{2}}}=\frac{-(1+y) \sqrt{1-y^{2}}}{1-y^{2}}=\frac{-\sqrt{1-y^{2}}}{1-y}=\frac{-x}{1-y}
\end{aligned}
$$

which indeed corresponds to $\frac{\Delta X}{\Delta Y}$ in the triangle in Fig. 2, as claimed. So, in this case too, the Leibnizian calculus handles the problem quite elegantly without any need for trigonometric functions. (In modern terms the differentiation of the integral $\int_{y}^{1} d y / \sqrt{1-y^{2}}$ is nothing but the derivative of the arccosine, but re-expressing it in such terms adds nothing of value or substance.)

These examples show that although the calculus of Leibniz and Newton is different from ours, it should not be assumed inferior. Modern calculus books leave us with the impression that "the only convenient way of representing a cycloid is by means of parametric equations" involving trigonometric functions, and so on for various other problems such as harmonic oscillation. Seeing, then, that Newton and Leibniz used other methods, one may be inclined to assume that their methods are inherently clumsier, and that these historical mathematicians would have recognized as much on the spot if we explained it to them; the only reason they didn't take this step must have been that they were conceptually limited by old ways of thinking, one naturally assumes. But the above examples suggest that the sense of superiority of the anachronistic modern perspective is hubristic. There are in fact ways in which the older approach can not only readily match what the modern approach can do, but even has a number of outright advantages over it. This suggests that Newton and Leibniz may very well have adhered to their style of calculus as a matter of conscious choice, with full awareness of the possibility of a more formula- or function-based approach.

In fact, there is even one intriguing bit of direct textual support for this interpretation. Although trigonometric formulas are completely absent from all calculus publications for several decades from the inception of the calculus onwards, there is one obscure early manuscript in which Newton does precisely what the hypothesis of 
a conceptual limitation postulated that he could not conceive: namely, he does use trigonometric formulas in calculus calculations, very much in the style of Euler or a modern textbook. In this manuscript from around 1680, Newton repeatedly writes " $f 1$ Cos A" - the fluxion of the cosine of the variable angle $A$ - in the course of one particular problem (MS Add.3963.7, 55r; MP.IV.459). Despite seemingly never working with such expressions before or since, Newton handles them effortlessly and incorporates them in his calculations without ado. It seems that Newton opted for this mode of expression in this particular problem because of its complexity; the more geometric phrasing he normally used (including in the other problems in this very manuscript) would be very verbose in this case, which would probably impede the clarity and overview of the calculations. Thus, Newton seems to have treated " $f 1$ Cos A" as a trivial shorthand. This shows that there was certainly no conceptual obstacle that held Newton back from using such expressions more extensively. Clearly, he realized that calculus could be built on such expressions, but consciously opted against it.

\section{Introduction of Trigonometric Functions by Euler}

Trigonometric functions were eventually introduced into the standard calculus repertoire by Euler. As late as 1754, this was still referred to as the "new calculus of sines" (Katz 1987, 316; Euler Opera 1.14.543). The context that led Euler (1739) to introduce this "new calculus" was the differential equation

$$
2 a \ddot{s}+\frac{s}{b}+\frac{a}{g} \sin \left(\frac{t}{a}\right)=0
$$

a periodically forced harmonic oscillator, whose solution is

$$
s(t)=D \cos \left(\frac{t}{\sqrt{2 a b}}\right)+C \sin \left(\frac{t}{\sqrt{2 a b}}\right)-\frac{a^{2} b \sin \left(\frac{t}{a}\right)}{g(a-2 b)},
$$

unless $2 b=a$, in which case

$$
s(t)=D \cos \left(\frac{t}{a}\right)+C \sin \left(\frac{t}{a}\right)+\frac{a t}{4 g} \cos \left(\frac{t}{a}\right) .
$$

Thus, unlike the simple harmonic oscillator that had long been handled by geometric paraphrase, the solutions of this differential equation involve combinations of trigonometric functions with different periods and trigonometric components multiplied by a nonconstant function (Fig. 3). The complexity of these solutions makes it very difficult to replace them with a geometrical description or to do without formulas of this type. As Euler put it, "there appear ... motions so diverse and astonishing that one is unable altogether to foresee until the calculation is finished" (Katz 1987, 318). Just as Newton had been led to use cosine formulas more 


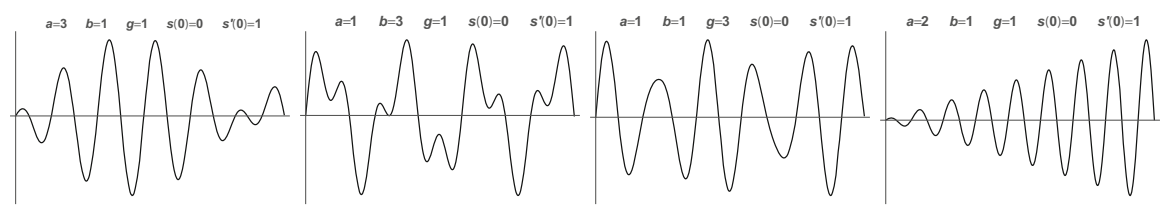

Fig. 3 Examples of solutions to the periodically forced harmonic oscillator considered by Euler (1739)

than half a century earlier in a particularly convoluted problem, so also to Euler the need to introduce such functions arises due to the complexity of the problem. In this way, it is possible to regard Euler's step not as a change in outlook, but merely as a consequence of the same priorities that were present even in Newton's time: the same outlook, consistently applied, leads to different styles in different contexts.

\section{$4 \quad$ Logarithmic Functions}

Just as trigonometric functions were absent from the early calculus, so was the $\operatorname{logarithm}$ function $\log (x)$. Despite explicitly dealing with the solution to the differential equation $\frac{d y}{d x}=\frac{a}{x}$ and referring to logarithmic curves by name in this connection, early calculus works by, for example, Leibniz (1684) and Johann Bernoulli (1692) refrain from using any kind of expression such as $\log (x)$ in a calculus setting. This could be interpreted as a shortcoming that hampered these works (Jahnke 2003, 111; Kowalewski 1914, 169). Interestingly, however, manuscript evidence shows that the reluctance to treat $\log (x)$ as one of the fundamental functions of the calculus cannot be attributed to an inability to conceive of the possibility of doing so. For Leibniz demonstrably did conceive of it years before, when he wrote "Log $y$ " in a calculus formula in a 1675 manuscript (AA.VII.5.325). Clearly, then, his decision not to write any formulas involving $\log (x)$ even when dealing with problems involving logarithms must be considered a conscious choice rather than unreflective adherence to older modes of expression.

Despite these notable early absences, the logarithm function entered official calculus discourse much sooner than trigonometric functions. The first published use of a logarithm expression in a calculus formula appears to be Leibniz (1694, 369), who writes "log. $x$." Despite never having used this notation before in print, Leibniz simply starts using it in media res without any indication that he has expanded the core repertoire of calculus functions, and with no qualms about taking its properties (such as "log. $x=\int d x: x$ ") for granted as if they were common knowledge. This makes sense if he regarded "log. $x$ " as a trivial shorthand for what was already well known in other (primarily verbal) terms.

Explicit uses of the logarithm function in calculus formulas did not catch on very quickly. Leibniz himself only used it passingly on one other occasion in print (Leibniz 1695, 314). Manfredi (1707), in what is effectively the first published integral calculus textbook, used the logarithm function quite extensively. Manfredi's 
notation is " $\mathrm{lx}$ " for $\log (x)$. Johann Bernoulli also used this notation on a number of occasions, yet as late as 1716 he still felt the need to explain that "by $l x$ I understand the logarithm of that $x$ " (Bernoulli 1716, 228). Thus, even two decades after its first appearance in print, Johann Bernoulli evidently considered the logarithm as a calculus function still not fully established as an elementary part of the standard vocabulary and notation of the calculus.

To conclude, trigonometric and logarithmic functions are seen as an indispensable part of calculus today, but historically the calculus was up and running for a decade before the logarithm function was used in a calculus context, and half a century before trigonometric functions followed suit. This was not for lack of occasion to use such functions: on the contrary, plenty of situations in which we would use these functions were encountered, and often explicitly recognized as concerning trigonometric or logarithmic relations. But this was expressed in verbal and geometric prose, rather than by explicit formulas.

From a modern point of view, this may strike us as potentially a sign of a cognitive limitation: perhaps these early practitioners of the calculus were inhibited by an older geometrical and prosaic paradigm of mathematical thought that prevented them from embracing the power of a more modern formal and purely analytic approach. If we knew only the published works of these mathematicians, then the historical record could be construed as fitting this hypothesis. After all, the calculus community relatively soon decided to favor the analytic style rather than the original approach.

Nevertheless, a strong case can be made that this anachronistically tempting hypothesis misses the mark. Manuscript evidence shows that the analytic approach - in the form of explicit use of trigonometric and logarithmic expressions in calculus formulas - was in fact considered by the creators of the calculus at a very early date, even though they generally opted against this approach in their published works. Indeed, one could argue that the approach they did take was the one best suited for their purposes. The strength of the modern analytic approach over the older style lies especially in handling complicated relationships with many components and parameters. So instead of seeing the transition from geometric to analytic expression as one from ignorance to enlightenment, one can interpret it as the natural outcome of sound preferences that remained consistent throughout: the analytic approach was introduced precisely when the occasion called for it - that is, when the efficiency and generality of formula-crunching outweighed the intuitive appeal of geometric expression.

\section{Dimensional Homogeneity}

Requiring equations to be dimensionally homogenous was another geometrically motivated choice persistent in the early calculus but at odds with later analytic practice (Bos 1974, 7). Thus a "length" such as $y$ cannot equal an "area" such as $x^{2}$, for instance, so one would rather write $a y=x^{2}$ to get a dimensionally balanced equation. This goes hand in hand with an emphasis on geometric interpretation 
rather than analytic formulas. Thus, for example, Johann Bernoulli, in his calculus lectures of 1692, writes the differential equation for the exponential curve as $y d x=a d y$, and when he separates the variables to $d x=a d y: y$ he then proceeds, for the sake of geometrical interpretation, to explicitly multiply by $a$ to get $a d x=a a d y: y$, which he then interprets visually as an equality of areas (Johann Bernoulli Opera III.421; Blåsjö 2017, 84). Bernoulli describes the resulting curve verbally and geometrically as a "Logarithmica," but has no symbolic notation for this at this time. By contrast, Euler, in his calculus textbook, happily works with dimensionally unbalanced equations such as $d y=d x / x$ (Euler 1755, § 180) and treats them purely analytically.

But again it would be a mistake to think of the geometric conservatism of the early calculus as a limitation of thought. Just as Leibniz privately used analytic notation for logarithm functions, so also in early manuscripts he was much more lax about working with equations violating dimensional homogeneity than in his later published works (Bos 1974, 12; Leibniz AA VII.5.xxxvi). A 1694 letter by Johann Bernoulli (Leibniz AA III.6.167) is explicit about what was probably widespread practice: working with a differential equation, he finds that a certain expression " $=d y$ " but then immediately adds: or for the sake of dimensional homogeneity " $=b d y$ " where $b$ is a new constant introduced solely to balance the equation dimensionally. That is to say, Bernoulli evidently had no problems working in an analytic mode that ignored formal geometric convention when expedient, only to then translate the end result into official form as a veritable afterthought. One finds the same type of reasoning in Leibniz's letters as well (Leibniz AA III.5.188).

This suggests that the convention of dimensional homogeneity was consciously adopted for the positive insights it could bring, without prejudice to other modes of reasoning. Indeed, dimensional analysis is a useful heuristic tool to this day (Pólya 1957, 202-205). Leibniz explicitly points out its usefulness as a check on calculations (AA VII.5.292). It was also very useful for purposes of geometric interpretation (the two examples from Bernoulli just mentioned are instances of this), which was often sensibly pursued in the early calculus as we have seen.

A related respect in which informal private practice was more flexible than formal expression was with regard to curve plotting. Impressively, accurate figures are quite common in the early calculus, but accompanying text is often couched in formal language quite aloof from the concrete, hands-on perspective that must have gone into producing the figures. Again, private manuscripts reveal a greater flexibility of thought than official discourse. Leibniz's description of the catenary $y=\left(e^{x}+e^{-x}\right) / 2$, for example, is in its published form rather stiltedly formulated in terms of the classical language of ratios. Privately, however, he operated with a more freewheeling numerical approach, including using a decimal representation of $e$ that he never revealed in print (Raugh and Probst 2019).

A similar example concerns how Jakob Bernoulli corrected his mistaken belief that the radius of curvature of any curve must be infinite at an inflection point. On intuitive grounds, Leibniz and Bernoulli had believed this to be a general principle. Indeed, it holds for simple inflection points, but there are exceptions. For instance, $x^{3}=y^{5}$ has an inflection point at the origin, yet the radius of curvature there is zero. 
This is because there are really multiple singularities in one at that point. Bernoulli deals with this by resolving the singularity. Instead of $x^{3}=y^{5}$, consider $x^{3}=y^{5}-b^{2} y^{3}$ for small $b$. This pulls the singularities apart. Now, the inflection point at the origin is simple again and the general rule holds: infinite radius of curvature. Bernoulli published this finding, but it is interesting to compare his manuscript notes with the published account (Bernoulli 1999, 151-154, 255-258). First of all, it is striking that Bernoulli writes his equations in dimensionally homogenous form $\left(a a x^{3}=y^{5}\right.$ and $\left.a a x^{3}=y^{5}-b b y^{3}\right)$ in his published article, but uses unbalanced equations $\left(x^{3}=y^{5}\right.$ and $\left.x^{3}=y^{5}-b b y^{3}\right)$ in his private notes. Furthermore, in his notes Bernoulli used detailed numerical calculations to plot the curve and explore its behavior. A table of $x$ and $y$ values, computed to two decimal places, occurs right next to the figure. This is suppressed or deemed unworthy of inclusion in the published version, where the figure appears without such supporting calculations and the formal presentation gives the impression that the conclusions were reached by abstract reasoning in eminently classical form. The look behind the scenes, at the numerical curve plotting and visual checks in Bernoulli's private notebook, reminds us once again that official published expression can give a misleading impression of the underlying thought process.

\section{Did Barrow Prove the Fundamental Theorem of Calculus?}

Isaac Barrow (1670) proved certain geometric theorems that could be interpreted as equivalents of the fundamental theorem of calculus. Barrow was writing before the introduction of the concepts and symbolism of derivatives and integrals. He speaks in purely geometrical terms, such as areas and tangents of curves. Should he nevertheless be regarded as having had an insight effectively equivalent to the fundamental theorem of calculus? Some have answered yes (Child 1916, vii, 31; Heath 1917, 133; Nauenberg 2014, 343), and seem to have judged that the direct translatability of Barrow's theorems into this modern form is in and of itself a compelling reason to accept this conclusion. At the other extreme, some emphasize precisely the geometric form and absence of symbolism as a conclusive reason to deny Barrow this insight (Bos 1980, 64-65; Wagner 2001; Sonar 2018, 55). It is advisable to steer clear of both of these extremes when trying to understand the meaning and significance of historical texts. Though these two interpretations are opposites, they share an excessive emphasis on the statement of theorem and proof in isolation. A sounder approach is to investigate how these theorems functioned in Barrow's thought as a whole, and how he saw them fitting into a bigger picture. Historians who have taken such a perspective have tended to came down against the claim that Barrow's theorems are equivalent to the fundamental theorem of calculus (Whiteside 1961, 367-368; Mahoney 1990, 236; Katz 2009, 539).

When taking this balanced approach, the historian can use modernized notation with benefit, as a tool for clarification and analysis, without falling into either of the opposite traps of the "mathematician's" anachronistic naiveté and the "historian's" knee-jerk rejection of all anachronistic devices. Let us take Barrow's relation to the 
Fig. 4 The subtangent $\sigma$ and subnormal $\eta$ of a given curve

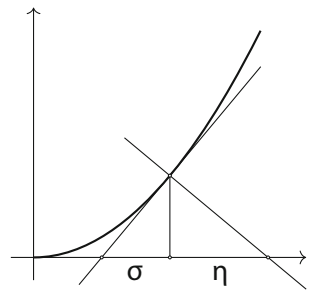

fundamental theorem of calculus as a case study illustrating this methodology. Thus, we shall first translate Barrow's results into modernized form, while remaining agnostic as to whether this is a faithful representation of his thought or not. This will enable us to see what the global organization of Barrow's text says about how he viewed the theorems in question.

The fundamental theorem of calculus has two parts:

$$
\begin{gathered}
\frac{d}{d t} \int_{a}^{t} y(x) d x=y(t) \\
\int_{a}^{b} y^{\prime}(x) d x=y(b)-y(a)
\end{gathered}
$$

By choosing the coordinate system so that $y(0)=0$ (which corresponds to Barrow's geometrical treatment), and using the notation $Y(x)=\int_{0}^{x} y(t) d t$, we can write this as

$$
\begin{gathered}
\frac{d}{d x} Y=y \\
\int_{0}^{x} y^{\prime}(t) d t=y(x)
\end{gathered}
$$

What we would interpret as results about derivatives, Barrow expresses in terms of subtangents and subnormals (i.e., the distance from the point where the tangent or normal cuts the axis to the point on the axis perpendicularly below the point on the curve in question; Fig. 4). In modernized language, the subtangent is $\sigma(y)=y / y^{\prime}$ and the subnormal is $\eta(y)=y y^{\prime}$.

The purported equivalent of FTC1 is Barrow's X.11. In our modernized form, it says $\sigma(Y)=Y / y$. Using the expression for the subtangent in terms of derivatives, we see that this is indeed equivalent to $Y^{\prime}=y$, or FTC1. The question is: is this theorem, in Barrow's mind, tied to the specific geometrical configuration, or is it a more structural and general result that it would be natural to call upon in any situation when we seek the rate of change of any anti-derivative or accumulation function? Barrow's own text - and in particular his treatment of a polar version of X.11 - gives us strong reason to favor the former rather than the latter interpretation, as follows. 
Fig. 5 The polar subtangent $\varsigma$ and polar subnormal $\nu$

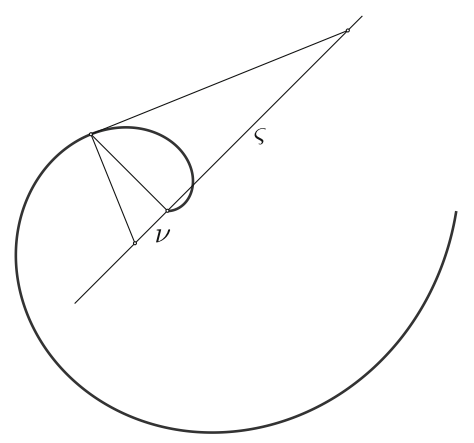

Throughout his treatise, Barrow interleaves results of the above form (in effect, assuming perpendicular rectilinear axes) with analogous results for curves defined in polar terms. In modernized form, $R(\theta)=\int_{0}^{\theta}(r(\vartheta))^{2} / 2 d \vartheta$ is the area-counting-function of the polar curve $r(\theta)$ (that is to say, $R(\theta)$ equals the area swept out by $r(\theta)$ from 0 to $\theta$ ). For polar curves, the subnormal and subtangent are defined not as segments of the $x$-axis, but as segments of the line perpendicular to the radial line through the origin point (Fig. 5). The polar subtangent is $\varsigma(r)=r^{2} / r^{\prime}$. The polar subnormal is $\nu(r)=r^{\prime}$.

Barrow's Proposition X.13 is a polar analog of X.11. It says: For the tangent at the point where $r(\theta)$ and $R(\theta)$ intersect, $\varsigma(R)=2$ (equivalently: $R^{\prime}=R^{2} / 2$ ). (Formally, the theorem only enables us to find the tangent at one particular point, but this is not an essential restriction. To find the tangent at another point on the curve $R(\theta)$, say where $R=k r$, we can let $\bar{r}=r / k$, and apply the theorem to the point of intersection of $\bar{r}(\theta)$ and $R(\theta)$, giving $\varsigma(R)=2 / k^{2}$.) Barrow proves X.13 from first principles, using the geometry of the polar differential triangle of $R(\theta)$. He presents X.13 as a "theorem of the same kind" as X.11, but he makes no use of the latter in the proof of X.13.

In modern terms, we could instead reason as follows. We know that $\varsigma(R)=R^{2} / R^{\prime}$ for any polar curve from the geometry of the polar subtangent. In this case, $R(\theta)$ is the area-counting function of $r(\theta)$, which means that $R(\theta)=\int_{0}^{\theta}(r(\vartheta))^{2} / 2 d \vartheta$. We can easily find its derivative using FTC1. It is $R^{\prime}(\theta)=r(\theta)^{2} / 2$. So the polar subtangent is $\varsigma(R)=2 R^{2} / r(\theta)^{2}$. In particular, when $R=r$, this simplifies to $\varsigma(R)=2$, which is Barrow's theorem X.13. This is a much shorter and easier proof than the elaborate deduction from first principles used by Barrow. The fact that Barrow did not use $\mathrm{X} .11$ to prove $\mathrm{X} .13$ in this way is thus quite compelling evidence that he lacked precisely that way of thinking about X.11 that warrants the FTC1 the epithet "fundamental."

Let us now turn to FTC2. In Lecture XI, Barrow presents "some Theorems ... relating to the Mensuration of Magnitudes by Tangents or Perpendiculars to Curves." That is to say, this lecture explains how to find areas in terms of tangents - or integrals in terms of derivatives. Indeed, we find a geometric equivalent of FTC2 in Barrow's XI.19. As in the case of FTC1, Barrow's statement and proof of 
Table 2 Results proved by Barrow (1670) in modernized form

\begin{tabular}{l|l}
\hline XI.1 & $\int \eta d x=y^{2} / 2$ \\
\hline XI.2 & $\int \eta y d x=y^{3} / 3$ \\
\hline XI.3 & $\int \eta y^{2} d x=y^{4} / 4$ \\
\hline & $\int \eta y^{3} d x=y^{5} / 5$ \\
\hline XI.4 & $\int Y y d x=Y^{2} / 2$ \\
\hline XI.5 & $\int \sqrt{Y} y d x=2 \sqrt{Y}^{3} / 3$ \\
\hline XI.7 & $\int \eta+x d x=\frac{1}{2}{\sqrt{x^{2}+y^{2}}}^{2}$ \\
\hline XI.10 & $\int \sigma d y=Y$ \\
\hline XI.12 & $\int \sigma y d y=\int y^{2} d x$ \\
\hline XI.13 & $\int \sigma y^{2} d y=\int y^{3} d x$ \\
\hline XI.16 & $\int \sigma^{2} d y=\int \sigma y d x$ \\
\hline XI.17 & $\int \sigma^{3} d y=\int \sigma^{2} y d x$ \\
\hline XI.19 & $\int y^{\prime} d x=y$ \\
\hline$X I .20$ & $\int\left(y^{\prime}\right)^{2} d x=\int y^{\prime} d y$ \\
\hline$X I .21$ & $\int\left(y^{\prime}\right)^{3} d x=\int\left(y^{\prime}\right)^{2} d y$ \\
\hline
\end{tabular}

Theorem XI.19 could quite reasonably be construed as corresponding directly to FTC2. Unless one takes a fundamentalist zero-tolerance hardline against any interpretation that transcends surface expression, there is nothing in Barrow's formulation of XI.19 that explicitly indicates any essential conceptual difference between it and FTC2. In other words, Barrow's XI.19 could be thought of as a full-fledged FTC2. But the more interesting question is: did Barrow think of it this way?

To answer this question, we must not focus on XI.19 itself, but rather look at the context in which this theorem occurs. Much as in the case of FTC1, a contextual perspective strongly suggests that Barrow did not think of XI.19 as fundamental or a conceptual cornerstone of the entire chapter. Rather, he seems to have viewed it as one particular geometrical result among many of a similar kind. Excluding polar curves, the area results that Barrow presents in Lecture XI are shown in Table 2.

In modern terms, it is trivial to verify these results by substituting the respective derivative expressions for $\eta$ and $\sigma$ and using FTC2 as well as basic "Leibnizian algebra" with differentials such as $\frac{d y}{d x} d x=d y$.

Today, we think of FTC2 as much more generic and prototypical than the other theorems in Barrow's Lecture XI. But in the geometrical language used by Barrow, it does not have an evidently exceptional status. XI.19 is not any more generic than XI.1 or XI.10 or even XI.7, which has a very direct geometrical meaning since $\eta+x$ is the distance from origin to end of subnormal and $\sqrt{x^{2}+y^{2}}$ is the distance from origin to the relevant point $(x, y)$ on the graph of $y(x)$.

Barrow proves these results by direct area considerations and not by verifying by differentiation that the right-hand sides are antiderivatives of the integrands, as we would in light of FTC2. He never uses XI.19 for such a purpose. There is no indication that he conceived of the possibility of taking XI.19 to be the centerpiece 
of the theory in this sense (or any other sense for that matter). Admittedly, XI.19 and its variations occur at the end of this sequence of results, which might suggest that it is the key result that crowns the entire development. Conceivably, Barrow might have perceived the fundamental nature of this result, yet opted to include and prove the previous theorems independently, perhaps for pedagogical, illustrative, or reference purposes. This possibility can be ruled out, however, by some revealing remarks later in the work.

In Appendix III to Lecture XII, Barrow offers a certain Theorem IV which he praises as "the most Fertile of all the Propositions foregoing. The greater Part of them being either contained in it, or deduced easily from it." The theorem in question may be considered a general result on change of variables. In slightly modernized terms, it states that if $y(x), g(x), h(y)$ are functions such that $\frac{\eta(y)}{y(x)}=\frac{h(y)}{g(x)}$ for all $x$ (which in modern terms is equivalent to $\left.g=y^{\prime} h\right)$ then $\int g d x=\int h d y$. Barrow notes the following special cases. If $h=1$, we get XI.19. If $h=y$, we get XI.1. If $y=g$, we get XI.10. From this Barrow concludes: "I cannot but accuse my Foresight, for not having first laid down this Theorem ... and then having deduced the rest from it, ... which I observe may be done." This very strongly suggests that Barrow did not see the possibility of using his FTC2-equivalent XI.19 to unify the entire sequence of results in Lecture XI. He expressly admits that he would have loved to give such a unified treatment if he had seen a way of doing so. Only later did he realize that this was in fact possible, and even then he still did not see XI.19 as fundamental but rather saw the unification as coming from another theorem altogether that does not correspond to the modern view of the centrality of the FTC.

Thus, in the case of Barrow's purported equivalents of both FTC1 and FTC2, Barrow misses major opportunities to use them to do the kind of work that FTC1 and FTC2 would do for us. This suggests that Barrow's theorems should not be considered equivalent to FTC1 and FTC2. The mere fact that Barrow used geometrical language does not in and of itself entail this conclusion. On the contrary, the early pioneers of the calculus often used geometrical language similar to that of Barrow, yet showed no signs of being constrained in their thinking when the occasion called for formal, nongeometrical applications of calculus principles abstractly in ways that are not visualizable in terms of tangents and areas (e.g., Engelsman 1984, Chap. 2). Hence a similar surface form of expression can be in one case indicative of a genuine conceptual limitation and in another case not. Rather than focusing on the surface form in which an idea is expressed, attention to its functional role in the author's broader argument is a better indicator of which aspects of the form of expression are incidental or essential to the underlying thought. Reconstructions in modernized mathematical terms can be a useful tool for such purposes. 


\section{Conclusion}

The early calculus used geometrical language and shunned the analytic style that came to predominate later. Many historians have tended to view this as a major conceptual divide (e.g., Speiser 2008, 108, 130). More mathematically inclined scholars, however, have been inclined to push back against this perspective and perceive more continuity in the underlying ideas than the surface form of expression would suggest (e.g., Fraser 2020, 198). The examples from Newton, Leibniz, and Bernoulli analyzed above weigh in favor of the mathematical interpretation. With regard to Barrow's purported geometrical version of the fundamental theorem of calculus, on the other hand, we have a reverse state of affairs. Mathematically inclined readers have been those most ready to accept the equivalence, while the historians' perception of conceptual discontinuity is borne out by closer analysis.

In the former case, the mathematician's intuition was best supported by the historian's methods: The mathematician's sense that the early practitioners of the calculus did not lack the ability to reason in a manner functionally equivalent to the modern analytic approach is borne out by textual evidence that they indeed explicitly used more analytic approaches in private manuscripts that in many cases have only recently been published through meticulous efforts of specialized historians. Conversely, in the Barrow case, the historian's intuition is arguably best supported by the mathematician's methods: A thoroughly modernistic reconstruction of Barrow's reasoning, far from being tantamount to a simplistic anachronistic fallacy, makes a compelling case against accepting the equivalence of Barrow's theorems and their modern counterparts. Efforts to understand past mathematical thought are well served by drawing on the strengths of each these diverse perspectives and approaches.

\section{$8 \quad$ Cross-References}

Christiaan Huygens: A XVIIth Century Mathematician Working in the Tradition of Archimedes and Apollonius

Descartes' Transformation of Greek Notions of Proportionality

- Heuristics and Mathematical Practice

- Historiography of Mathematics

\section{References}

Barrow I (1670) Lectiones geometricae: in quibus (praesertim) generalia curvarum linearum symptomata declarantur, London. Reprinted in William Whewell (ed) The mathematical works of Isaac Barrow. Cambridge University Press, 1860, 155-316. English translations in Barrow (1735) and Child (1916)

Barrow I (1735) Geometrical lectures: explaining the generation, nature and properties of curve lines. Stephen Austen, London 
Bernoulli J (1999) Die Werke von Jakob Bernoulli, Bd. 5. Differentialgeometrie, edited by David Speiser, André Weil, \& Martin Mattmüller. Birkhäuser, Basel

Bernoulli J (1692) Lectiones Mathematicae de Methodo Integralium, aliisque. In: Bernoulli J (ed) Opera III, pp 385-558. Partial German translation in Kowalewski (1914)

Bernoulli J (1716) Problema: data serie linearum per rectae in eadem Linea constantis variationem prodeunte invenire aliam seriem linearum, quarum quaevis priores omnes ad angulos rectos secabit. Acta Eruditorum:226-230

Blåsjö V (2017) Transcendental curves in the Leibnizian calculus. Elsevier, Oxford, United Kingdom

Bos HJM (1974) Differentials, higher-order differentials and the derivative in the Leibnizian calculus. Arch Hist Exact Sci 14(1):1-90

Bos HJM (1980) Newton, Leibniz and the Leibnizian tradition, chapter 2. In: Grattan-Guinness I (ed) From the Calculus to set theory, 1630-1910. Princeton University Press, Princeton

Child JM (1916) The geometrical lectures of Isaac Barrow. Open Court Publishing, Chicago and London

Engelsman SB (1984) Families of curves and the origins of partial differentiation, Elsevier NorthHolland mathematics studies, vol 93

Euler L (1739) De novo genere oscillationum. Commentarii academiae scientiarum Petropolitanae 11:128-149. (presented 1739). Opera Omnia, Series 2, vol 10, 78-97. E126, 1750

Euler L (1755) Institutiones calculi differentialis, Saint Petersburg

Fraser C (2020) Review of Shank (2018). J Mod Hist 92(1):197-198

Heath TL (1917) Review of Child (1916). Math Gaz 9(130):131-134

Jahnke HN (2003) A history of analysis. American Mathematical Society. Providence, Rhode Island, USA

Katz VJ (1987) The Calculus of the trigonometric functions. Hist Math 14(4):311-324

Katz VJ (2009) A history of mathematics: an introduction, 3rd edn. Addison-Wesley

Kowalewski G (1914) Die erste Integralrechnung: Eine Auswahl aus Johann Bernoullis Mathematischen Vorlesungen über die Methode der Integrale und anderes, Ostwald's Klassiker der exakten Wissenschaften 194. Engelmann, Leipzig \& Berlin

Leibniz GW (1684) Nova methodus pro maximis et minimis, itemque tangentibus, quae nec fractas, nec irrationales quantitates moratur, \& singulare pro illis calculi genus. Acta Eruditorum:467473

Leibniz GW (1694) Constructio propria problematis de Curva Isochrona Paracentrica. Acta Eruditorum:364-375

Leibniz GW (1695) Responsio ad nonnullas difficultates, a Dn. Bernardo Niewentiit circa methodum differentialem seu infinitesimalem motas. Acta Eruditorum:310-316

Leibniz GW (AA) (1923-present ongoing) Sämtliche Schriften und Briefe, Gottfried-WilhelmLeibniz-Gesellschaft, Academie Verlag, leibnizedition.de

Mahoney MS (1990) Barrow's mathematics: between ancients and moderns. In: Feingold M (ed) Before Newton: the life and times of Isaac Barrow. Cambridge University Press, Cambridge

Manfredi G (1707) De constructione aequationum differentialium primi gradus, Bologna

Nauenberg M (2014) Barrow, Leibniz and the geometrical proof of the fundamental theorem of the Calculus. Ann Sci 71(3):335-354

Newton I (MP) (1967-1981) The mathematical papers of Isaac Newton, 8 vols, edited by D. T. Whiteside. Cambridge University Press, Cambridge, pp 1967-1981

Pólya G (1957) How to solve it, 2nd edn. Princeton University Press, Princeton

Raugh M, Probst S (2019) The Leibniz catenary and approximation of $e-$ an analysis of his unpublished calculations. Hist Math 49:1-19

Simmons GF (1996) Calculus with analytic geometry, 2nd edn. McGraw-Hill, New York, USA

Sonar T (2018) The history of the priority dispute between Newton and Leibniz. Birkhäuser, Basel, Switzerland

Speiser D (2008) Discovering the principles of mechanics 1600-1800, edited by Kim Williams \& Sandro Caparrini. Birkhäuser 
Wagner J (2001) Barrow's fundamental theorem. Coll Math J 32(1):58-59

Whiteside DT (1961) Patterns of mathematical thought in the later seventeenth century. Arch Hist Exact Sci 1(3):179-388 